\title{
The Second Wave of Covid-19 and the Present and Continuing Challenges of the Health and Care System from the Side of a Numerical Analysis
}

\author{
Mariam Almahdi Mohammed Mu'lla, ${ }^{1,2}$, Munirah Oudah Alanazi1 \\ ${ }^{1}$ Department of Mathematics, University of Hafr Al-Batin (UoHB), Hafr Albatin, KSA \\ ${ }^{2}$ University of Kordofan, El-Obeid, North Kordofan, Sudan \\ Email: marimdx2014@gmaial.com, halaplus85@gmail.com
}

How to cite this paper: Mu'lla, M.A.M. and Alanazi, M.O. (2021) The Second Wave of Covid-19 and the Present and Continuing Challenges of the Health and Care System from the Side of a Numerical Analysis. Open Access Library Journal, 8: e7665.

https://doi.org/10.4236/oalib.1107665

Received: June 20, 2021

Accepted: July 26, 2021

Published: July 29, 2021

Copyright $\odot 2021$ by author(s) and Open Access Library Inc.

This work is licensed under the Creative Commons Attribution International License (CC BY 4.0).

http://creativecommons.org/licenses/by/4.0/

\begin{abstract}
The second wave of Covid-19 presents new and ongoing challenges to the health and care system. The main objective of this study is to take into account the quality of health work and health guidelines in anticipation of the health care systems not collapsing, thus saving more time in the fight against this epidemic and appropriate preparation for each emergency situation arising from this epidemic. In this study we will deal with Covid19 in terms of mathematics, to look at this epidemic from multifaceted aspects. We want to continue to support leaders and health and care workers through the next phase of the pandemic in a scientific and thoughtful way. The secretions and resources we produce and make sure are scientific and useful study. Let's find out how we can continue to provide helpful support. Avoiding the causes that led to the outbreak of coronavirus disease 2019 (Covid-19) through the study, we found that the focus is on the basic measure of the number of beds in the hospital and the possibility of spacing for patients. Treatment beds must be provided for patients at risk of organ failure. Patients are provided with an intensive care unit bed (also known as an intensive care unit bed).
\end{abstract}

\section{Subject Areas}

Mathematical Analysis, Modern Mathematical Algebra, Foundations of Mathematics, And the Field of Health and Care

\section{Keywords}

Public Health Emergency of International Concern, Modeling the Epidemiology, SEIR Model, SEIR Dynamics, Endemic Equilibrium, 
Qualitative Analysis and Stability

\section{Introduction}

In this study, this paper will address the second wave of Covid19 in some countries of the world including Sudan, and the elderly are affected by this wave. Coronavirus disease 2019 (COVID-19) outburst were seen in Wuhan city of China in December 2019 caused by a novel member of coronavirus family, i.e., Severe Acute Respiratory Distress Syndrome Coronavirus-2 (SARS-COV-2) [1] [2]. Over the course of the current period, we have heard a range of perspectives from people who have channeled their scientific and medical knowledge about Covid-19 through a mix of written and audio content. International Committee on Taxonomy of Viruses (ICTV) officially named it as SARS-COV-2 [3]. This study targets leaders working in the field of health, social care or public health in gathering places in universities, schools, and the voluntary and independent sectors. We want to know the challenges you face, and help us avoid the risks and impacts of this pandemic, through our study, we have found that it is very clear that the cure rate and mortality rate are directly related to whether or not healthcare capacity has been exceeded.

In China, from 3 January 2020 to 11:32am CEST, 7 July 2021, there have been 119,036 confirmed cases of COVID-19 with 5554 deaths, reported to WHO. As of 26 June 2021, a total of 1,189,495,322 vaccine doses have [2].

As it was the time of the spring festival, visitors have come from various parts of China, so it spread in a different parts of China [3] [4] In our model, we assumed that it was important to seriously consider this obvious fact, cure rate and disease-affected mortality rate were considered to be variable according to health care capacity status with fixed low limits.

1) With the use of real-time Reverse Transcriptase-Polymerase chain reaction (RT-PCR) researcher identified it caused due to SARS-COV-2 [4].

2) The cases increased in large number, so the World Health Organization (WHO) declared COVID-19 a Public Health Emergency of International Concern (PHEIC), i.e. Pandemic, in 11-March, 2020.

3) The study of genome sequences of SARS-COV-2 revealed that its genome is $79.5 \%$ similar to SARS-COV and $96 \%$ to bat SARS-COV [5].

We will use epidemiological modeling of the COVID-19 outbreak using a SEIR model with four compartments, namely, exposed groups, infectious groups, and recovered groups, and the proportion of prevalence for each group controlled by a set of numerically ODE and PDEs. There is modeling of the spread of Covid-19 with travel data and infection data but it is incomplete and we are looking at how to complete. The outbreak of the novel coronavirus disease (COVID-19) in 2019 began to occur and escalate during a special holiday period in China, and it is likely spread through the seafood market, which also includes wildlife like bats, snakes and other poisonous and polluting animals. And the 
actual spread began for several reasons

1) A large volume of travel occurred between Chinese cities, then around the world, which led to the spread of the disease on a wide scale.

2) Failure to take precautionary measures in multiple regions in the countries of the world, for example Italy, France, Spain and the Maghreb, then Sudan moved Egypt, the Persian Gulf and Saudi Arabia to all over the world.

\subsection{Travel Procedures Integrated Data SEIR Model}

We are modeling the epidemiology of the COVID-19 outbreak using the SEIR model with Four compartments, groups exposed, nfectious, and recovered, and the prevalence ratio for each group governed by a set of numerically normal differential equations [6],

$$
S=-\frac{\beta S I}{N}, \quad E=+\frac{\beta S I}{N}-\alpha E, \quad I=+\alpha E-\gamma I, \quad R=+\gamma I
$$

The transmission rates between the four groups, $\beta, \alpha$ and, are the reflections of the contact duration $B=1 / \beta$, the passive or static period $A=1 / \alpha$, the infectious period $C=1 / \gamma$, and $N=S+E+I+R$ is About the total population. We interpret the latent rate $\alpha$ and the infection rate as being related to a disease of COVID-19, and assume that they are constants in all European Union countries. [7] [8] We explain the connection rate $\beta=\beta(t)$ as a specific behavior for this virus, and we assume that it varies with each country in terms of the rate of population and the rate of closeness between people and it may vary over time and time to reflect the effect of the precautionary, societal and political measures [9] [10]. To facilitate interpretation, we express the contact rate between $\beta(t)=R(t) / C$ in terms of the time-variable effective continuous reproduction number $R(t)$ [6]. For effective reproduction and reproduction number, we assume the type of hyperboloid tangent ansatz,

$$
R(t)=\left[R_{0}-\frac{1}{2}\left[1+\tanh \left[t-t^{*}\right] / T\right]\right]\left[R_{0}-R_{t}\right] .
$$

Ansatz ensure a fast and smooth transition from $R_{0}$ at the start of the virus outbreak to Current reproduction number $R_{t}$ under travel restrictions and procedures Closing countries, where $t$ is the adaptation time and $T$ is the transition time.

With the description and assumptions mentioned above, the established model, which takes into account the effect of the level of availability of care opportunities provided by the health care system for individuals of various classifications, HIV-positive and non-infected, with a time delay representing a latent period before the onset of symptoms is as follows: [8] [9]

$$
\begin{gathered}
\frac{d S}{d t}=b-\beta S(t) I(t)-d S(t), \\
\frac{d E}{d t}=\beta S(t) I(t)-\gamma \tilde{E}(t, \tau)-\delta E(t)-d E(t),
\end{gathered}
$$




$$
\begin{gathered}
\frac{d I}{d t}=\gamma \tilde{E}(t, \tau)-\left[\mu_{1}+\mu_{2}(1-C(t))\right] I(t)-\left[\alpha_{1}+\alpha_{2} C(t)\right] I(t)-d I(t), \\
\frac{d R}{d t}=\left[\alpha_{1}+\alpha_{2} C(t)\right] I(t)+\delta E(t)-d R(t),
\end{gathered}
$$

The model of equations $d S, d E, d I, d R$ and parameters (unknown) $\alpha_{i}, \beta_{i}, \gamma_{i}, \delta_{i}$.

$$
\begin{gathered}
d S_{i}(t)=-\frac{\beta_{j}(t)}{N_{j}^{S}} I_{j}(t) S_{j}(t)-\frac{\alpha_{j}(t)}{N_{j}^{S}} E_{j}(t) S_{j}(t) \\
+\sum_{i=1}^{N}\left(\frac{S_{i}(t) m_{i j}(t)}{P_{i}(t)}\right)-\frac{S_{j}(t) \sum_{i=1}^{N} m_{j i}(t)}{P_{j}(t)} \\
d E_{i}(t)=-\frac{\beta_{i}(t)}{N_{i}^{E}} I_{i}(t) E_{i}(t)-\frac{\alpha_{i}(t)}{N_{i}^{E}} E_{i}(t) S_{i}(t) \\
-k_{j}(t) E_{i}(t)+\sum_{i=1}^{N}\left(\frac{E_{i}(t) m_{i j}(t)}{P_{i}(t)}\right)-\frac{E_{i}(t) \sum_{i=1}^{N} m_{j i}(t)}{P_{j}(t)} \\
d I_{j}(t)=k_{j}(t) E_{i}(t)-\gamma_{i}(t) I_{j}(t) \\
+k_{I}\left(\sum_{i=1}^{N}\left(\frac{I_{i}(t) m_{i j}(t)}{P_{i}(t)}\right)-\frac{I_{j}(t) \times \sum_{i=1}^{N} m_{j i}(t)}{P_{j}(t)}\right) \\
d R_{j}(t)=\gamma_{j}(t) I_{j}(t)
\end{gathered}
$$

\subsection{The Problem of Information Disappearance, Non-Appearance or Loss}

One of the main reasons for the rapid spread of viruses:

The lack of awareness of the delayed information depends on the real and urgent situation of the population and the most prominent time and lack of speed in response to the information in the number of confirmed cases reported, as it depends on the ability of the particular country or city to conduct the examination and tests in addition to the potential bureaucracy in the local reporting system and its speed.

Problem of Information Latency and loss, one reason for rapid spread of virus:

- Delay information: insufficient awareness of the real situation of the community

- The reason for most of the observed information delay is the number of confirmed cases reported, which depend on the ability and testing capabilities of the particular country or city, as well as the potential bureaucracy in the local reporting system and the speed of reporting.

$$
I>C \text { or } I \gg C
$$

Model: The first problem is summarized by the integration of travel data, transportation, mixing, and crowding. The second problem is summarized in the time of transmission of information to the responsible authorities [7].

\subsection{Review of SEIR Model}

On day $t$, there are 
Susceptible $S_{i}(t)$, Exposed $E_{i}(t)$, Infected $I_{i}(t)$, Recovered $R_{i}(t)$

City $i$ with population $=P_{i}$

Eventual percentage of infection $=\delta_{i}$

\subsection{Basic SEIR Dynamics}

System dynamics We will use epidemiological modeling of the outbreak of COVID-19 using the SEIR model that shows the four sections, namely, the exposed, infectious, and recovered groups, and the prevalence rate for each group using a set of equations:

For a single population $S(t)=-\beta S(t) I(t)$

(network), we can put $E(t)=\beta S(t) I(t)-\kappa E(t)$

The state equations as $I(t)=\kappa E(t)-\gamma I(t) \& R(t)=\gamma I(t)$

$$
\Rightarrow\left\{\begin{array}{l}
\Delta S(t)=-\beta S(t-1) I(t-1) \\
\Delta E(t)=\beta S(t-1) I(t-1)-\kappa E(t-1) \\
\Delta I(t)=\kappa E(t-1)-\gamma I(t-1) \\
d R(t)=\gamma I(t-1)
\end{array}\right.
$$

The outbreak of the new Coronavirus disease (COVID) for the year 2019 began to occur and escalate during a special holiday period in China (about more than 20 days surrounding the year 2019 to the year 2020 and then extended to 2021), which occurred during the first of 2019, a large volume of travel between cities, Which led to the outbreak of the disease in multiple regions in China, then it moved to the rest of the world, then in the second period 2020 most countries worked to close their airports in order to avoid the spread of the disease, and then the airports were opened in the year 2020 conditional on examining the traveler for the disease and in the third period 2020-2021 appeared a new type of virus developed in Britain and South Africa, and the countries worked again to take health precautions and mathematical algebraic variables were chosen to study this case, on day $t$, there are: Susceptible $S_{i}(t)$, Exposed $E_{i}(t)$, Infected $I_{i}(t)$, Recovered $R_{i}(t)$, City $i$ with population $=P_{i}$, Eventual percentage of infection $=\delta_{i}$.

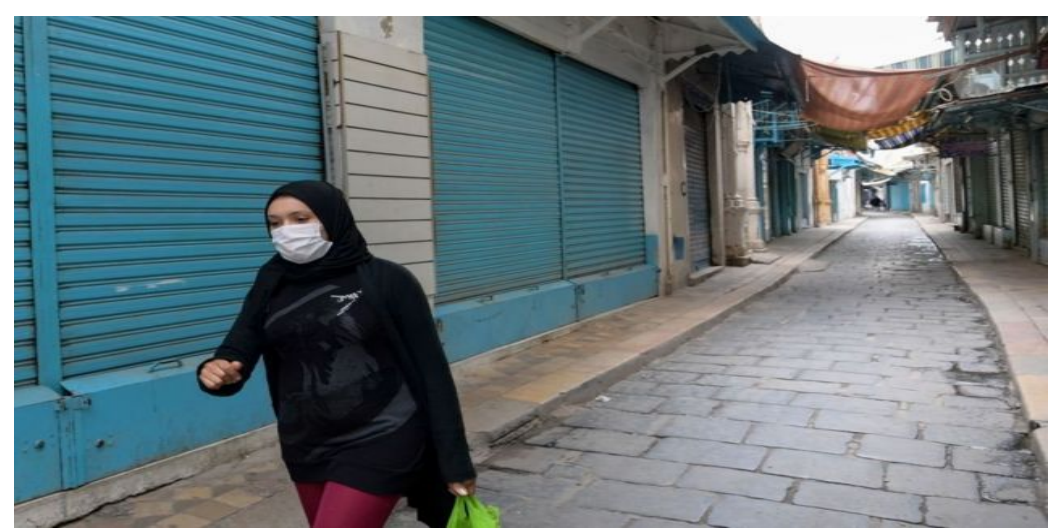

This picture was taken from the Internet during the first week of February 
2021, showing the health precautions taken in some cities in the world.

\section{The Model, Its Qualitative Analysis and Stability}

We consider an epidemic model that designed SEIR with an arrangement of compartments consisting of $(S)$ vulnerable to COVID-19 disease, $(E)$ susceptible to COVID-19 and infected individuals $(I)$ recovered individuals $(R)$ of the total population $(N)$. All parameters used in the model are non-negative constants parameters $b$ and $d$ represent birth rate and natural death rate of all compartments, respectively. In the model, it is assumed that all newborns are included to the susceptible compartment. Also $\beta$ is effective contact rate between susceptible and infectious individuals $\gamma$ is progression rate of exposed individuals who are not infectious to class $R$. The function $c(t)$, which takes value in the interval of $[0,1]$, represents the level of availability to opportunities provided by health care system to individuals who are become infectious at time $t . \alpha_{1}$ is natural recovery rate of the infectious class, that is, this value is minimum recovery rate for the model. Also $\alpha_{2} c(t)$ is contribution rate to recovery rate of health care system. Thus clearly $\alpha_{1}+\alpha_{2}$ is maximum recovery rate. Furthermore, $\mu_{1}$ is minimum disease induced death rate and $\mu_{2}(1-c(t))$ is a factor increasing the disease induced deaths due to cannot benefit ion from health care system. So, it is clear that $\mu_{1}+\mu_{2}$ is maximum death rate. As it can be estimated, the case that $c(t)$ tends to zero means that all hospital opportunities are almost consumed away, the case of $c(t)=1$ says that all hospital opportunities can be used fully. [11] [12] Additionally the $N(t), S(t), E(t), I(t), R(t)$ describe the number of individuals in the relevant compartment at time $t$ These variables are clearly nonnegative and $N(t)=S(t)+E(t)+I(t)+R(t)$ for all $t \geq 0$. With above descriptions and assumptions, the model, which is taken into account the effect of level of availability to opportunities provided by heath care system to individuals, with a time delay representing a latent period is as follows are:

$$
\begin{gathered}
\frac{d S}{d t}=b-\beta S(t) I(t)-d S(t), \\
\frac{d E}{d t}=\beta S(t) I(t)-\gamma \tilde{E}(t, \tau)-\delta E(t)-d E(t), \\
\frac{d E}{d t}=\beta S(t) I(t)-\gamma \tilde{E}(t, \mu)-\delta E(t), \\
\frac{d I}{d t}=\gamma \tilde{E}(t, \mu)-\left(\mu_{1}+\mu_{2}(1-c(t))\right) I(t)-\left(\alpha_{1}+\alpha_{2} c(t)\right) I(t)-d I(t), \\
\frac{d R}{d t}=\left(\alpha_{1}+\alpha_{2} c(t)\right) I(t)+\delta E(t)-d R(t),
\end{gathered}
$$

where the evolution of the density in the exposed individuals given by:

$$
\left(\frac{\partial}{\partial t}+\frac{\partial}{\partial \mu}\right) \tilde{E}(t, \mu)=-d \tilde{E}(t, \mu)
$$

where $\tilde{E}(t, 0)$ the number of exposed individuals to coronavirus at the time $t$, 
and $\tilde{E}(t, 0)=\beta S(t) I(t)$.

From its solution we obtain the Equations:

$$
\tilde{E}(t, \mu)=\beta S(t-\mu) I(t-\mu) e^{-d \mu}
$$

Therefore, the system (7) can be rearranged as follows:

$$
\begin{gathered}
\frac{d S}{d t}=b-\beta S(t) I(t)-d S(t) \\
\frac{d E}{d t}=\beta S(t) I(t)-\gamma \beta S(t-\mu) I(t-\mu) e^{-d \mu}-(d+\delta) E(t), \\
\frac{d I}{d t}=\gamma \beta S(t-\mu) I(t-\mu) e^{-d \mu}-\left(\mu_{1}+\mu_{2}(1-c(t))\right) I(t) \\
-\left(\alpha_{1}+\alpha_{2} c(t)\right) I(t)-d I(t), \\
\frac{d R}{d t}=\left(\alpha_{1}+\alpha_{2} c(t)\right) I(t)+\delta E(t)-d R(t),
\end{gathered}
$$

And also we find the periods that must be taken into account the impact of the level of availability of opportunities provided by the health care system to individuals on a regular basis, because a time delay represents the latent period that is shown as follows:

$$
\begin{gathered}
\frac{d S}{d t}=A-d_{1} S-\lambda \frac{S I}{S+I}+r I, \\
\frac{d I}{d t}=\lambda \frac{S I}{S+I}-\left(d_{2}+r\right) I,
\end{gathered}
$$

where $S(t)$ and $I(t)$ denote the number of susceptible and infectvie individuals at time $t$, respectively. $A$ is the recruitment rate of the population, $d_{1}$ is the natural death rate of the population, $d_{2}$ is the death rate for individuals infected with the COVID-19 disease which includes the natural death rate and disease-related death rate, $r$ is the cure rate for infected individuals, $\lambda$ is the standard incidence rate. Has the fundamental scattering number $R_{0}=\frac{\lambda}{d_{2}+r}$ and if $R_{0} \leq 1$ the injury-free equilibrium $E_{0}\left(\frac{A}{d}, 0\right)$ of model (1) is not globally stable, and if $R_{0}>1$ then the endemic equilibrium $E_{*}\left(S^{*}, I^{*}\right)$, may be locally stable, we obtain the following COVID-19 discrete time for the Lemma (2.1).

Lemma (2.1)

Let $R_{0}=\frac{\lambda}{d_{2}+r}$ (the basic reproductive rate), and we have the following result as regards the existence of the equilibria.

1) If, $R_{0} \leq 1$, then we have only the COVID-19 disease-free equilibrium $E_{1}^{*}\left(\frac{A}{d_{1}}, 0\right)$.

2) If $R_{0}<1$, then we have two equilibria: the COVID-19 disease-free equilibrium $E_{1}^{*}\left(\frac{A}{d_{1}}, 0\right)$ and the endemic equilibrium $E_{2}^{*}\left(S^{*}, I^{*}\right)$ where 


$$
\begin{aligned}
& S^{*}=\frac{A\left(d_{2}+r\right)}{d_{1}\left(d_{2}+r\right)+d_{2}\left(\lambda-d_{2}-r\right)}, \\
& I^{*}=\frac{A\left(\lambda-d_{2}-r\right)}{d_{1}\left(d_{2}+r\right)+d_{2}\left(\lambda-d_{2}-r\right)}
\end{aligned}
$$

Now, we study the stability of equilibria $E_{1}^{*}$ and $E_{2}^{*}$, Then the Jacobian matrix at the equilibrium $E^{*}\left(S^{*}, I^{*}\right)$ is

$$
J\left(E^{*}\right)=\left(\begin{array}{cc}
1-h\left[d_{1}+\lambda \frac{I^{* 2}}{\left(S^{*}+I^{*}\right)^{2}}\right] & h\left[r-\lambda \frac{S^{* 2}}{\left(S^{*}+I^{*}\right)^{2}}\right] \\
h \lambda \frac{I^{* 2}}{\left(S^{*}+I^{*}\right)^{2}} & 1+h\left[\lambda \frac{S^{* 2}}{\left(S^{*}+I^{*}\right)^{2}}-\left(d_{2}+r\right)\right]
\end{array}\right)
$$

The corresponding characteristic equation of $J\left(E^{*}\right)$ can be written as

$$
\omega^{2}-\operatorname{tr} J\left(E^{*}\right) \omega+\operatorname{det} J\left(E^{*}\right)=0
$$

After simple computing, we obtain the local stability result of the disease-free equilibrium $E_{1}^{*}\left(\frac{A}{d_{1}}, 0\right)$, which is shown in the following.

\section{Lemma (2.2)}

1) $E_{1}^{*}\left(\frac{A}{d_{1}}, 0\right)$ is a sink if $0<h<\min \left\{\frac{2}{d_{1}}, \frac{2}{d_{2}+r-\lambda}\right\}$,

2) $E_{1}^{*}\left(\frac{A}{d_{1}}, 0\right)$ is a source if $h>\max \left\{\frac{2}{d_{1}}, \frac{2}{d_{2}+r-\lambda}\right\}$,

3) $E_{1}^{*}\left(\frac{A}{d_{1}}, 0\right)$ is non-hyperbolic if $h=\frac{2}{d_{1}}$ or $\frac{2}{d_{2}+r-\lambda}<h<\frac{2}{d_{1}}$,

4) $E_{1}^{*}\left(\frac{A}{d_{1}}, 0\right)$ is a saddle if $\frac{2}{d_{1}}<h<\frac{2}{d_{2}+r-\lambda}$ or $\frac{2}{d_{2}+r-\lambda}<h<\frac{2}{d_{1}}$,

On the local stability of equilibrium $E_{2}^{*}\left(S^{*}, I^{*}\right)$ we have the following result

Lemma (2.3)

1) $E_{2}^{*}\left(S^{*}, I^{*}\right)$ is a sink if one of the following conditions holds:

i) $\Delta \geq 0$ and $0<h<h_{1}$,

ii) $\Delta<0$ and $0<h<h_{3}$,

2) $E_{2}^{*}\left(S^{*}, I^{*}\right)$ is a source if one of the following conditions holds:

i) $\Delta \geq 0$ and $h>h_{2}$,

ii) $\Delta<0$ and $h>h_{3}$

3) $E_{2}^{*}\left(S^{*}, I^{*}\right)$ is non-hyperbolic if one of the following conditions holds:

i) $\Delta \geq 0$ and $h=h_{1}$ or $h_{2}$,

ii) $\Delta<0$ and $h=h_{3}$,

4) $E_{2}^{*}\left(S^{*}, I^{*}\right)$ is a saddle if the following conditions holds:

i) $\Delta \geq 0$ and $h_{1}<h<h_{2}$,

Where $h_{1}$ and $h_{2}, h_{3}$ is: 


$$
\begin{gathered}
h_{1}=\frac{\lambda\left(\lambda-d_{2}-r+d_{1}\right)-\lambda \sqrt{\Delta}}{\left(\lambda-d_{2}-r\right)\left[d_{1}\left(d_{2}+r\right)+d_{2}\left(\lambda-d_{2}-r\right)\right]}, \\
h_{2}=\frac{\lambda\left(\lambda-d_{2}-r+d_{1}\right)+\lambda \sqrt{\Delta}}{\left(\lambda-d_{2}-r\right)\left[d_{1}\left(d_{2}+r\right)+d_{2}\left(\lambda-d_{2}-r\right)\right]}, \\
h_{3}=\frac{\lambda\left(\lambda-d_{2}-r+d_{1}\right)}{\left(\lambda-d_{2}-r\right)\left[d_{1}\left(d_{2}+r\right)+d_{2}\left(\lambda-d_{2}-r\right)\right]}, \\
\Delta=\left(d_{2}+r-\lambda-d_{1}\right)^{2}-\frac{4\left(\lambda+d_{2}-r\right)\left[d_{1}\left(d_{2}+r\right)+d_{2}\left(\lambda-d_{2}-r\right)\right]}{\lambda} .
\end{gathered}
$$

The proofs of Lemma (2.2) and Lemma (2.3) are simple and hence we omit them. From the above discussion we find that if condition (i) in conclusion (3) of Lemma (2.2) holds, then one of the two eigenvalues of the matrix $J\left(E^{*}\right)$ is -1 and the other is neither 1 nor -1 . We can rewrite conditions (i) in the following form: [13] [14]

$$
\left(A, d_{1}, d_{2}, r, h, \lambda\right) \in M_{1} \cup M_{2}
$$

where

$$
\begin{aligned}
& M_{1}=\left\{\left(A, d_{1}, d_{2}, r, h, \lambda\right): h=h_{1}, A>0, \Delta \geq 0, R_{0}>1,0<d_{1}, d_{2}, r, \lambda\right\} \\
& M_{2}=\left\{\left(A, d_{1}, d_{2}, r, h, \lambda\right): h=h_{2}, A>0, \Delta \geq 0, R_{0}>1,0<d_{1}, d_{2}, r, \lambda\right\}
\end{aligned}
$$

In the following section we will see that there may be a flip bifurcation round equilibrium $E_{2}^{*}\left(S^{*}, I^{*}\right)$ if $h$ varies in the small neighborhood of $h_{1}$ or $h_{2}$ and $\left(A, d_{1}, d_{2}, r, h_{1}, \lambda\right) \in M_{1}$ or $\left(A, d_{1}, d_{2}, r, h_{2}, \lambda\right) \in M_{2}$. When condition (ii) in conclusion (3) of Lemma (2.2) holds, we can see that the two eigenvalues of the matrix $J\left(E^{*}\right)$ are a pair of conjugate complex numbers, the modules of which are 1. Condition (ii) can be written in following form:

$$
\left(A, d_{1}, d_{2}, r, h, \lambda\right) \in N \text {, }
$$

where

$$
N=\left\{\left(A, d_{1}, d_{2}, r, h, \lambda\right): h=h_{3}, A>0, \Delta \geq 0, R_{0}>1,0<d_{1}, d_{2}, r, \lambda\right\} .
$$

\section{Proposition 2.3}

The set

$$
\Omega=\left\{i=\left(i_{1}, i_{2}, \cdots, i_{n}\right) \mid i_{k} \in N \text { and } \int_{0}^{\infty} i_{k}(a) d a \leq 1 \text { for } k \in \mathbb{N}_{n}\right\}
$$

is a positively invariant set of equation.

We only need to consider the equation with initial conditions in $\Omega$.

The following results tells us that if there is initial infection then the infection persists.

$$
\left\{\frac{\partial i_{k}(t, a)}{\partial t}+\frac{\partial i_{k}(t, a)}{\partial a}=-\gamma(a) i_{k}(t, a), k \in \mathbb{N}_{n}\right\}
$$




$$
i_{k}(t, 0)=\sigma k\left(1-\int_{0}^{\infty} i_{k}(a) d a \odot(i(t, .))\right),
$$

By the model formulation and Proposition (2.3), we only need to consider Equation (15) with initial conditions in the following result tells us that if there is initial infection in COVID-19 then the infection persists [14].

Where $(i(t,))=.\sum_{k=1}^{n} k p(k) \int_{0}^{\infty} \frac{\beta(a) i_{k}(t, a)}{\langle k\rangle}$, and $\langle k\rangle=\sum_{k=1}^{n} k p(k)$

Since $S_{k}(t)+\int_{0}^{\infty} i_{k}(t, a)=1$, in this equation equivalent to (15)

$$
\left\{\begin{array}{l}
\frac{d S_{k}(t)}{d t}=-k \sigma S_{k}(t) \odot(i(t, .))+\int_{0}^{\infty} \gamma(a) i_{k}(t, a) d a \\
\frac{\partial i_{k}(t, a)}{\partial t}+\frac{\partial i_{k}(t, a)}{\partial a}=-\gamma(a) i_{k}(t, a) \\
i_{k}(t, 0)=k \sigma S_{k}(t) \odot(i(t, .))
\end{array}\right.
$$

Then Equation (15) is well-posed. Moreover, it is not difficult to show the following result [12] [15] [16].

\section{Conclusion}

In fact, the interest in manufacturing and taking a drug or vaccine that has an accurate and direct effect in combating the COVID-19 pandemic caused by the new type of coronavirus that is spreading at the speed of fire, has led to all countries. Epidemiological modeling of the COVID-19 outbreak was used by the four-section SEIR model, the groups exposed, infectious, recovered, and the prevalence rate for each group controlled by a set of ODE and PDE equations numerically. Of the world adopting policies aimed at curbing the spread of COVID-19, in order not to exceed the number of patients currently infected and to increase critical cases inside hospitals, which calls for raising the capacity of health care in them. With these policies, the main objective of this study is to take into account the quality of health work and health guidelines in anticipation of the health care systems not collapsing, thus saving more time in combating this epidemic and adequate preparation for every emergency that arises from this epidemic ( $\alpha_{1}$ and $\mu_{1}$, respectively). Thus, through our study, the disease is expected to increase in some countries of the world when the level of availability of quality health care and the opportunities provided by the health care system decrease. In the appendices is a list of countries by availability of hospital beds per 1000 or 100,000 people, as published by local governments, the international organization (OECD, European Union), from academic or other sources. The number of beds available in health facilities per person is an important indicator of a country's health care system [1]. And we found through the study that the focus is on the basic measure of the number of beds in the hospital, which are divided and occupied differently. Classic hospital beds also called Dedicated treatment beds for patients at risk of organ failure, patients are provided with an intensive care unit bed (also known as an intensive care unit bed) or intensive care unit (CCB) beds [15] [16]. 


\section{Conflicts of Interest}

The authors declare no conflicts of interest.

\section{References}

[1] Shereen, M.A., Khan, S., Kazmi, A., Bashir, N. and Siddique, R. (2020) COVID-19 Infection: Emergence, Transmission, and Characteristics of Human Coronaviruses. Journal of Advanced Research, 24, 91-98. https://doi.org/10.1016/j.jare.2020.03.005

[2] Docherty, A.B., et al. (2020) Features of 20133 UK Patients in Hospital with Covid-19 Using the ISARIC WHO Clinical Characterisation Protocol: Prospective Observational Cohort Study. BMJ, 369, m1985. https://doi.org/10.1136/bmj.m1985

[3] Lovato, A. and de Filippis, C. (2020) Clinical Presentation of COVID-19: A Systematic Review Focusing on Upper Airway Symptoms. Ear, Nose \& Throat Journal, 99, 569-576. https://doi.org/10.1177/0145561320920762

[4] Siordia Jr., J.A. (2020) Epidemiology and Clinical Features of COVID-19: A Review of Current Literature. Journal of Clinical Virology, 127, Article ID: 104357. https://doi.org/10.1016/j.jcv.2020.104357

[5] Wang, L.S., Wang, Y.R., Ye, D.W. and Liu, Q.Q. (2020) A Review of the 2019 Novel Coronavirus (COVID-19) Based on Current Evidence. International Journal of Antimicrobial Agents, 12, 1-7.

[6] Linka, K., Peirlinck, M., Sahli Costabal, F. and Kuhl, E. (2020) Outbreak Dynamics of COVID-19 in Europe and the Effect of Travelrestrictions. Computer Methods in Biomechanics and Biomedical Engineering, 23, 710-717. https://doi.org/10.1080/10255842.2020.1759560

[7] https://combatcovid.hhs.gov/i-have-covid-19-now/monoclonal-antibodies-high-ris k-covid-19-positive-patients?msclkid=0ec22c26133c13b290824c9db3e67da516

[8] Khan, M.A. and Atangana, A. (2020) Modeling the Dynamics of Novel Coronavirus (2019-ncov) with Fractional Derivative. Alexandria Engineering Journal, 59, 2379-2389. https://doi.org/10.1016/j.aej.2020.02.033

[9] Ivorra, B., Ferrandez, M.R., Vela-Perez, M. and Ramos, A.M. (2020) Mathematical Modeling of the Spread of the Coronavirus Disease 2019 (COVID-19) Considering Its Particular Characteristics: The Case of China. Communications in Nonlinear Science and Numerical Simulation, 88, 105303. https://doi.org/10.1016/j.cnsns.2020.105303

[10] Ndairou, F., Area, I., Nieto, J. and Torres, D.F.M. (2020) Mathematical Modeling of COVID-19 Transmission Dynamics with a Case Study of Wuhan. Chaos, Solitons and Fractals, 135, 109846. https://doi.org/10.1016/j.chaos.2020.109846

[11] Diekmann, O., Heesterbeek, J.A.P. and Metz, J.A.J. (1990) On the Definition and the Computation of the Basic Reproduction Ratio $R_{0}$ in Models for Infectious Diseases in Heterogeneous Populations. Journal of Mathematical Biology, 28, 365-382. https://doi.org/10.1007/BF00178324

[12] Diekmann, O. and Heesterbeek, J.A.P. (2000) Mathematical Epidemiology of Infectious Diseases: Model Building, Analysis and Interpretation. John Wiley and Sons, Chichester.

[13] Lakshmikantham, S., Leela, S. and Martynyuk, A.A. (1989) Stability Analysis of Nonlinear Systems. Marcel Dekker, Inc., New York, 220. https://doi.org/10.1142/1192

[14] Ma, Z., Zhou, Y. and Wang, W. (2004) Mathematical Models and Studies in Epi- 
demic Dynamics. Kexue Publication, Beijing.

[15] https://covid19.who.int/region/wpro/country/cn

[16] https://en.wikipedia.org/wiki/list of countries by hospital beds 